\title{
Expression of ovate family protein 8 affects epicuticular waxes accumulation in Arabidopsis thaliana
}

\author{
Yao Tang ${ }^{1}$, Wei Zhang ${ }^{2}$, Yan-Li Yin ${ }^{1}$, Peng Feng ${ }^{1}$, Hong-ling Li ${ }^{3}$ and Ying Chang ${ }^{1 *}$ (])
}

\begin{abstract}
Background: Transcription factors could regulate multiple aspects of plants growth and development, which is significant to plants. Ovate family proteins (OFPs) that are named due to contain OVATE domain, a 70-AA C-terminal conserved domain from the protein OVATE gene encodes, are plant-specific transcription factors family. Some members of OFPs have been shown to function as transcription factors to regulate plant growth and development, but little is known about the function of AtOFP8.

Results: Here, we found AtOFP8 maybe involve in transcriptional regulation of the epicuticular waxes in Arabidopsis thaliana. First, we observed that the distribution of epicuticular waxes of wild type plants was more than that of Atofp8-1 deletion mutants, but less than that of 35S:HA-AtOFP8 transgenic overexpression lines not only on the leaves but also on the stems utilizing scanning electron microscopes. Second, we extracted waxes from leaves and stems of three types of plants respectively to measure the waxes content and composition by gas chromatography-mass spectrometer (GC-MS), and the results of the total content of waxes were consistent with the results of scanning electron microscopes. Finally, we found that the expression of 12 genes related to the synthesis and metabolism of waxes was changed in the Atofp8-1 mutants and 35S:HA-AtOFP8 transgenic lines compared with wild type plants.
\end{abstract}

Conclusions: These findings suggest that AtOFP8 could change the accumulation of epicuticular waxes.

Keywords: AtOFP8, Epicuticular waxes, Transcription factor

\section{Background}

Ovate family proteins (OFPs) that are named due to contain OVATE domain, a 70-AA C-terminal conserved domain from the protein OVATE gene encodes, are plantspecific family of regulatory proteins (Hackbusch et al. 2005; Liu et al. 2002). All the time, we think that there are 18 genes that are predicted to encode proteins containing OVATE domain in Arabidopsis thaliana (Wang et al. 2007). It was not until 2014 that we knew that there are 19 OFPs genes in A. thaliana rather than 18 (Liu et al. 2014). A. thaliana ovate family proteins (AtOFPs) have been proved to be a novel transcriptional factor family, which could control multiple aspects of plant growth

*Correspondence: 2427472516@qq.com

${ }^{1}$ Northeast Agricultural University, Harbin 150030, China

Full list of author information is available at the end of the article and development (Wang et al. 2011). So far, only a few members of AtOFPs have been shown to function as transcription factors to regulate plant growth and development (Li et al. 2011; Pagnussat et al. 2007; Wang et al. 2007). AtOFP1 was shown to regulate cell elongation by suppressing the expression of Gibberellin 20 oxidase 1 (GA20ox1), a gene encoding the key enzyme in gibberellin (GA) biosynthesis (Wang et al. 2007). AtOFP5 was reported to negatively regulate the activity of a BELL-like homeodomain protein 1-knotted1-like homeodomain protein 3 (BLH1-KNAT3) complex during early embryo sac development (Pagnussat et al. 2007). AtOFP4 was demonstrated to participate in the regulation of secondary cell wall formation by interacting with KNAT7 ( $\mathrm{Li}$ et al. 2011). However, little is known about the function of AtOFP8. 
As we all know, epicuticular waxes are complex mixtures of very long chain fatty acids (VLCFAs) and their derivatives, which are significant to plants (Shepherd and Griffiths 2006). They could form an important protective layer to protect the internal structure of the plant body (Koch and Ensikat 2008). Except control the loss of water by stomatal transpiration, plants also reduce the water loss by epicuticular waxes, which plays a crucial role in resisting water stress (Sieber et al. 2000). Waxes layer on the surface of the leaves has reflection effect to ultraviolet rays and light, which can protect the leaves from the dangers of radiation (Holmes and Keiller 2002). Until now, lots of genes related to the biosynthesis and metabolism of epicuticular waxes have been reported. LTP4, a gene of lipid transfer proteins gene family, could bind to fatty acids and transfer phospholipids between the membranes (Arondel et al. 2000). CER2 gene expressed in an organ- and tissue-specific manner affects epicuticular waxes accumulation and the stems of cer2 mutants are bright green (Xia et al. 1996). CER4 encoded an alcohol-forming fatty acyl-coenzyme A reductase (FAR) is responsible for the synthesis of primary alcohols, which involves in epicuticular waxes production (Rowland et al. 2006). FAR4 could affect the formation of primary alcohols and the encoded enzyme could increase the length of the fatty acid chain (Domergue et al. 2010). Researchers generally believe that if stems or leaves have a glossy green phenotype, it is indicative of waxes deficiency, and conversely, if plants have whitish green stems, it is most likely displayed more waxes (Go et al. 2014).

During our cultivation of Arabidopsis, we observed that 35S:HA-AtOFP8 transgenic lines had whitish green leaves and Atofp8-1 mutants were lighter green than wild-type plants. According to this phenomenon, we guessed the function of AtOFP8 maybe associate with the epicuticular waxes. To testify whether transcription factor AtOFP8 could affect epicuticular waxes accumulation and how to change epicuticular waxes content and composition, we had verified from multiple perspectives. In our study, we reported the difference of the shape and the content of epicuticular waxes by comparing Atofp 8 1 mutants, wild-type plants and 35S:HA-AtOFP8 transgenic lines. Moreover, the expression of 12 genes related to the biosynthesis and metabolism of waxes was significant difference among them. These results suggest transcription factor AtOFP8 could change epicuticular waxes content and composition, perhaps by changing genes expression.

\section{Materials and methods}

\section{Plant materials and growth conditions}

Arabidopsis thaliana (Columbia-0 ecotype) was used as wild-type plants in our experiment. 35S:HA-AtOFP8 transgenic lines and Atofp 8-1 mutants were previously reported (Wang et al. 2011). All seeds were planted in the 1/2 Murashige and Skoog (MS) medium containing 0.8\% agar and 3\% sucrose and transferred into the soil a week later. Plants were grown under long-day conditions (14 h light $/ 10 \mathrm{~h}$ dark cycles) at $23^{\circ} \mathrm{C}$.

\section{Scanning electron microscopy}

The stems and the rosette leaves of 5-week-old plants were chosen to dry in the oven at $50^{\circ} \mathrm{C}$ after fixing on the foam board. Then they were plated by gold membrane on the surface of the samples glued to the sample table using ion sputtering coating machine and epicuticular waxes were observed using a scanning electron microscope.

\section{Transmission electron microscopy}

The stems and the rosette leaves of 5-week-old plants were chosen to fix in a solution containing $2.5 \%$ glutaraldehyde in $0.1 \mathrm{~mol} \mathrm{~L}^{-1}$ phosphate buffer, $\mathrm{pH} 6.8$, at $4{ }^{\circ} \mathrm{C}$ for $4 \mathrm{~h}$. The samples were then rinsed in $0.1 \mathrm{~mol} \mathrm{~L}^{-1}$ phosphate buffer, $\mathrm{pH} 6.8$, three times and further fixed in $1 \%$ osmium tetroxide for $3 \mathrm{~h}$ at $4{ }^{\circ} \mathrm{C}$. After rinsing with $0.1 \mathrm{~mol} \mathrm{~L}^{-1}$ phosphate buffer at normal temperature, the samples were dehydrated and embedded in Spurr's resin. After processing embedded block, thin sections $(60 \mathrm{~nm}$ thickness) were prepared with an ultramicrotome. After staining, the sections were observed under a transmission electron microscope.

\section{GC-MS analysis}

$150 \mathrm{mg}$ of the stems and the rosette leaves of 5-week-old plants were weight to put into small beakers respectively and $5 \mathrm{~mL}$ of chloroform were added into small beakers for $1 \mathrm{~min}$ at room temperature. After adding $5 \mu \mathrm{g}$ of $\mathrm{C} 24$ into the extracted chloroform solvent as internal reference, the solvent was transferred into GC bottles and evaporated under a gentle stream of nitrogen. Then $10 \mu \mathrm{L}$ bis(trimethylsilyl)trifluoroacetamide (BSTFA) and $10 \mu \mathrm{L}$ pyridine were added into the GC bottles to redissolve waxes samples and the waxes mixtures were heated for $1 \mathrm{~h}$ at $70{ }^{\circ} \mathrm{C}$. Samples were measured by injecting $1 \mu \mathrm{L}$ of sample and helium $\left(2 \mathrm{~mL} \mathrm{~min}^{-1}\right)$ was used as a carrier gas during which the oven temperature was increased from 80 to $290{ }^{\circ} \mathrm{C}$ at a rate of $4{ }^{\circ} \mathrm{C} / \mathrm{min}$ and finally $30 \mathrm{~min}$ at $290^{\circ} \mathrm{C}$.

\section{Gene chip analysis}

When the plants grew up to 3 weeks old, leaves were cut off and sent to Shanghai Bohao Biotechnology Corporation to make agilent chip to find differentially expressed genes. All chip results were analyzed by SBC analysis system (SAS), an expression microarray data analysis software of this corporation. 
Table 1 qRT-PCR primer sequence

\begin{tabular}{|c|c|c|}
\hline Gene & Primer name & Primer sequence $\left(5^{\prime}-3^{\prime}\right)$ \\
\hline \multirow[t]{2}{*}{ LTP4 } & LTP4-F & CACCTCCGTGCTGTGCAG \\
\hline & LTP4-R & TGGCGCAGTTGGTGCTC \\
\hline \multirow[t]{2}{*}{ At2g18370 } & At2g18370-F & GCATCAAGTCAGTGGCTAATAGTGT \\
\hline & At2g18370-R & CATTCGCCTACATATATTATACGGC \\
\hline \multirow[t]{2}{*}{ CER4 } & CER4-F & TTGATGCTGTTTCTGATGTGATGC \\
\hline & CER4-R & ACGACCTTCCCTTCTTTGTTGG \\
\hline \multirow[t]{2}{*}{ KCS2 } & KCS2-F & GGTTGTAGTGCTGGTCTTATCTCC \\
\hline & KCS2-R & GGACGGTGTGGATGAGTTGG \\
\hline \multirow[t]{2}{*}{$\mathrm{ADH} 1$} & $\mathrm{ADH} 1-\mathrm{F}$ & ATGCCTTCAAGACTCATCCGA \\
\hline & $\mathrm{ADH} 1-\mathrm{R}$ & TTGATTTCCGAGAATGGCACT \\
\hline \multirow[t]{2}{*}{ KCS10 } & KCS10-F & CTCGACGTCAGTTTAATGCATCT \\
\hline & KCS10-R & GGGTTCTTGGTAGCGTATTCGT \\
\hline \multirow[t]{2}{*}{ FAR4 } & FAR4-F & CACAATGGCTCAGTTCAGTTTCTAC \\
\hline & FAR4-R & CGCAGTTTCTCAGTATTCGTATCG \\
\hline \multirow[t]{2}{*}{ GL2 } & GL2-F & TAGAGATGAAGCTCGTCGGCAT \\
\hline & GL2-R & GTTGCCTCTGTCTTGTCCCTT \\
\hline \multirow[t]{2}{*}{ DFR } & DFR-F & GGTTACTTTGTTCGTGCCACC \\
\hline & DFR-R & CCTCAGATAAATCAGCCTTCC \\
\hline \multirow[t]{2}{*}{ At2g42990 } & At2g42990-F & GCCTCGAAAAGATGTGAAGAG \\
\hline & At2g42990-R & TGAATTCTTAACACGCCAGAGG \\
\hline \multirow[t]{2}{*}{ At1g06360 } & At1g06360-F & GACCCACATAGCCCTATCGAA \\
\hline & At1g06360-R & TGCTTCAAGTCCATCACGTT \\
\hline \multirow[t]{2}{*}{ CER2 } & CER2-F & CGAGCCGTCTACTTCTTCAAGG \\
\hline & CER2-R & GTTGCAGCGAATGTAAGGTATGG \\
\hline \multirow[t]{2}{*}{ ACT2 } & $\mathrm{ACT} 2-\mathrm{F}$ & CCAGAAGGATGCATATGTTGGTGA \\
\hline & ACT2-R & GAGGAGCCTCGGTAAGAAGA \\
\hline
\end{tabular}

Table 2 The transcription level of AtOFP8 in 35S:HAAtOFP8 overexpression plants and Atofp8-1 mutant

\begin{tabular}{lllll}
\hline Locus & Symbol & Accession & $\begin{array}{l}\text { FC in 35S:HA- } \\
\text { AtOFP8 }\end{array}$ & FC in Atofp8-1 \\
\hline AT5G19650 & OFP8 & NC_003076 & 323.1614 & -13.7830
\end{tabular}

\section{Quantitative real-time RT-PCR analysis}

RNA of 3-week-old plants leaves was extracted using RNA extraction kit according to the manufacturer's instructions. Reverse transcription (RT) was performed according to reverse transcription kit instructions to synthesize first-strand cDNA. Quantitative RT-PCR reactions were performed using SYBR green fluorescent quantitation PCR kit. The PCR primers used were listed in Table 1 . The values for each set of primers were normalized relative to the $A C T I N 2$ gene. qRT-PCR reactions were performed in biological triplicates using RNA samples extracted from three independent replicate samples. The comparative $\Delta \Delta \mathrm{C}_{\mathrm{T}}$ method was used for the calculation of the results.

\section{Chlorophyll leaching assays}

When the plants grew up to 5 weeks old, 1 g of rosette leaves was weight and put into erlenmeyer flasks wrapped with aluminum foil. Then $30 \mathrm{~mL}$ of $80 \%$ ethanol were poured into erlenmeyer flasks. Remove $3 \mathrm{~mL}$ solution from erlenmeyer flasks to measure absorbance at 647 and $664 \mathrm{~nm}$ using a spectrophotometer as the amount of extracted chlorophylls every $10 \mathrm{~min}$. The solution using for measuring was back into erlenmeyer flasks every time. The absorbance measured after $24 \mathrm{~h}$ was as the total amount of chlorophylls. The calculation of chlorophyll content was based on the method previously (Lolle et al. 1998).

\section{Water loss assays}

The rosette leaves of 5-week-old plants were cut out and put into water for $1 \mathrm{~h}$ in the dark to make water contents be same. After drying the water on the surface of leaves, the leaves were weight using a microbalance every $20 \mathrm{~min}$. The water loss rate (v) was calculated by the formula:

$$
\mathrm{V}=\left(\mathrm{W}_{0}-\mathrm{W}_{1}\right) / \mathrm{W}_{0} \times 100 \%
$$

Where $\mathrm{W}_{0}$, weight of leaves before dehydration; $\mathrm{W}_{1}$, weight of leaves after dehydration.

\section{Results}

\section{Identification of plant materials}

35S:HA-AtOFP8 transgenic plants and Atofp $8-1$ mutant were previously reported (Wang et al. 2011). Gene chip results showed that the transcription level of AtOFP8 in 35S:HA-AtOFP8 overexpression plants increased more than 300 -fold, while decreased by nearly 14 -fold in the Atofp8-1 mutant (Table 2).

\section{Leaves are whitish green in 35S:HA-AtOFP8}

After transplanting plants in the soil, we gradually found there were significant differences in the color and phenotype of leaves among them. Although there was no significant difference in the phenotype of leaves between Atofp8-1 and Col-0, leaves of 35S:HA-AtOFP8 were thicker and flatter than that of Col-0. Moreover, leaves of 35S:HA-AtOFP8 were more whitish green than that of Col-0, whereas leaves in Atofp8-1 were lighter green than that of Col-0 (Fig. 1). We guessed this phenomenon might be related to epicuticular waxes.

\section{The distribution of epicuticular waxes is thin in Atofp8-1, whereas dense in 35S:HA-AtOFP8}

Depending on the difference in the color and phenotype of leaves, we guessed this was most likely caused by the different distribution of epicuticular waxes. In order to verify whether this was related to the distribution of 


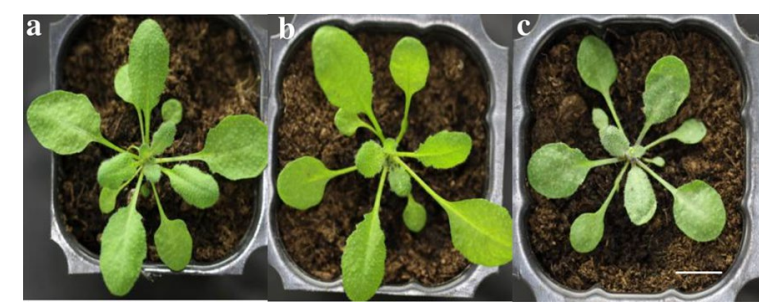

Fig. 1 Image of seedlings 25 days old. a Col-0, b Atofp8-1, c 35S:HAAtOFP8. Bar $=1 \mathrm{~cm}$

epicuticular waxes, surface of stems and leaves were observed respectively using the scanning electron microscope. On the surface of stems, the morphology of epicuticular waxes in Atofp 8-1, Col-0 and 35S:HA-AtOFP8 are clearly different each other. Moreover, epicuticular waxes in Col-0 seem to lie more densely than that in Atofp 81, but not so densely as the 35S:HA-AtOFP8 (Fig. 2a). Changes in content were the same in the leaves (Fig. 2b). Then we did transmission electron microscopy analysis to leaves and stems of Atofp 8-1, Col-0 and 35S:HA-AtOFP8. The results showed that there were no significant differences in the thickness of the cuticle in neither leaves nor stems (Fig. 3a, b). These observations imply that AtOFP8 conld only affect the distribution of epicuticular waxes without changing the cuticle waxes.
The content of waxes is reduced in Atofp8-1, while increased in 35S:HA-AtOFP8

To verify further whether waxes content was changed, GC-MS was used to measure the content in the stems and leaves respectively. Although the content of waxes was not changed exponentially, there was still some difference among the totals of waxes. Results showed that the totals of waxes were $387.56,356.05$ and $412.23 \mu \mathrm{g} \mathrm{g}^{-1}$ respectively in the stems of Col-0, Atofp $8-1$ and 35S:HAAtOFP8. Compared with the wild type plants, the total content of waxes of Atofp8-1 was slightly lower, while the total content of waxes in 35S:HA-AtOFP8 transgenic plants was slightly higher (Fig. 4a). The different components of the stems waxes also changed. The content of primary alcohols and ketone in 35S:HA-AtOFP8 transgenic plants was significantly higher than that in wild type, while Atofp $8-1$ did not change significantly with wild type (Fig. 4a). However, the totals of waxes in the leaves were almost approximate (Fig. $4 \mathrm{~b}$ ), but there were distinct variations between the specific components. In the leaves of the 35S:HA-AtOFP8, alkanes were significantly increased compared with the wild type plants, while primary alcohols were significantly reduced (Fig. 4c, d). These results indicate that AtOFP8 could change the content and composition of waxes. $\mathbf{a}$

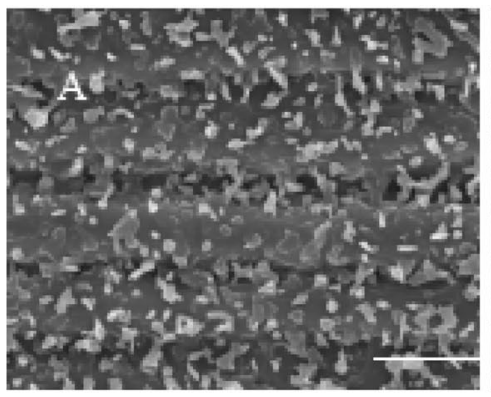

b

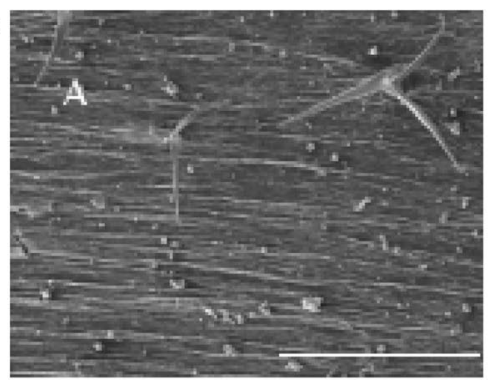

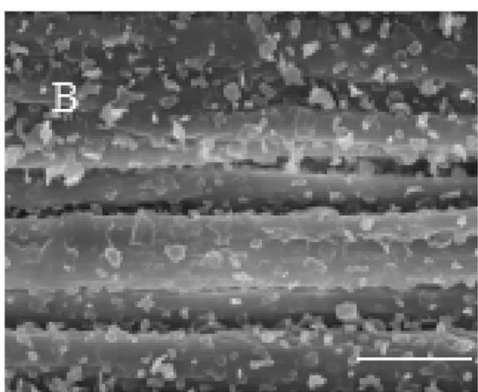
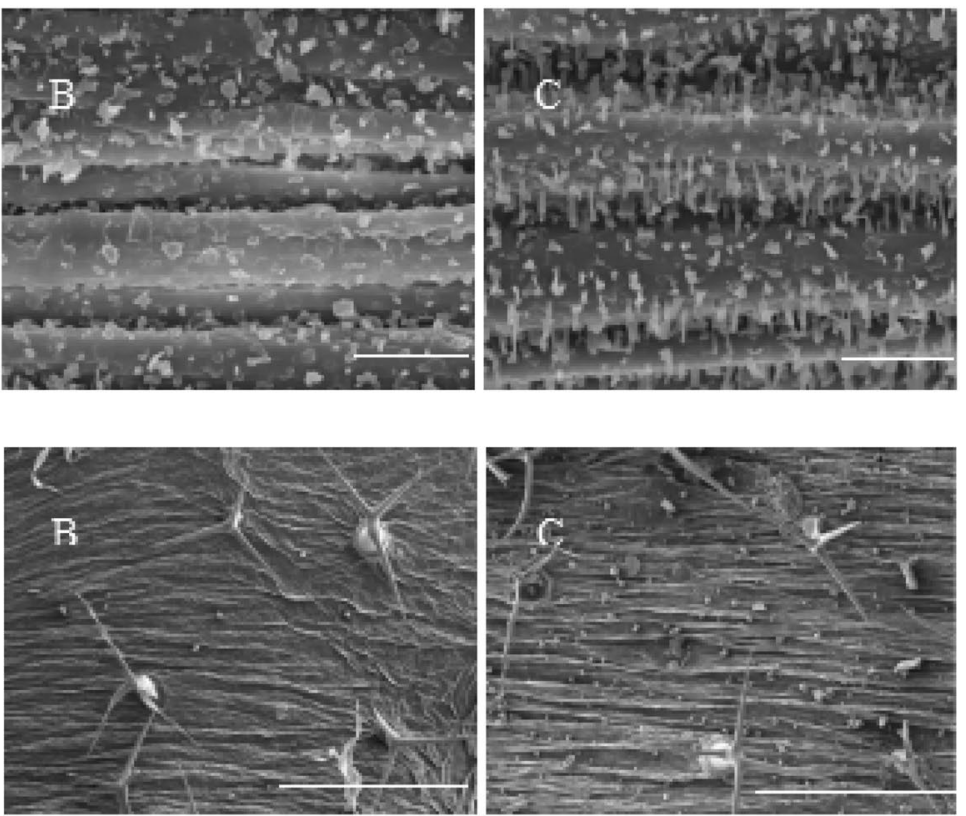

Fig. 2 Scanning electron microscopy images of epicuticular waxes. a Images of the stems in the Col-O (A), Atofp8-1 (B) and 35S:HA-AtOFP8 (C). Bars $=10 \mu \mathrm{m}$. b Images of the leaves in the Col-0 (A), Atofp8-1 (B) and 35S:HA-AtOFP8 (C). Bars $=10 \mu \mathrm{m}$ 
$\mathbf{a}$

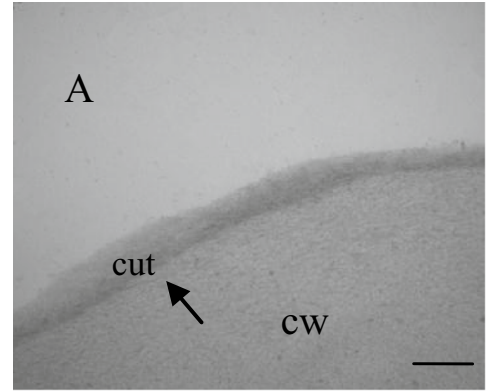

b

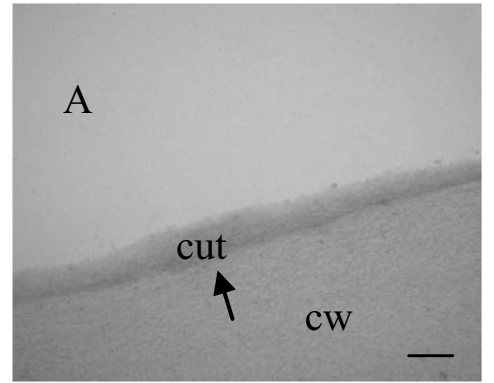

B

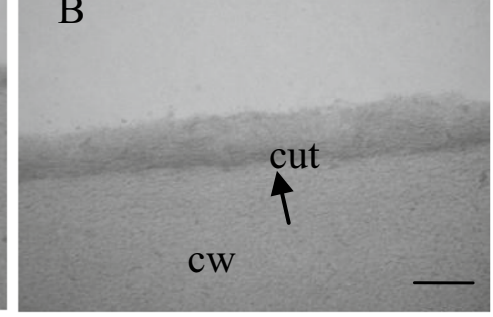

B

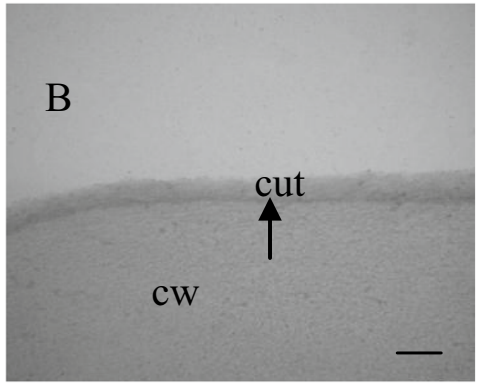

$\mathrm{C}$

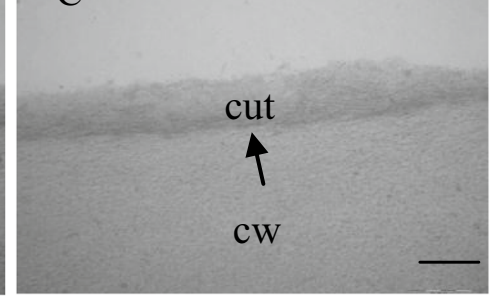

$\mathrm{C}$

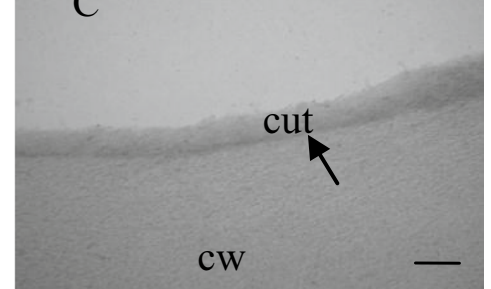

Fig. 3 Transmission electron microscopy images of epicuticular waxes. cut cuticle, cw cell wall. a Images of the stems in the Col-0 (A), Atofp8-1 (B) and 35S:HA-AtOFP8 (C). Bars $=200 \mathrm{~nm}$. b Images of the leaves in the Col-O (A), Atofp8-1 (B) and 35S:HA-AtOFP8 (C). Bars $=200 \mathrm{~nm}$

\section{Gene chip results show that AtOFP8 affects many aspects of Arabidopsis growth and development}

To find out which genes caused by the change of epicuticular waxes, we made gene chip analysis among the Atofp 8-1, 35S:HA-AtOFP8 and Col-0. Results showed that the expression of abundant genes was changed in the Atofp 8-1 and 35S:HA-AtOFP8 compared with Col-0. FC (35S:HA-AtOFP8 vs Col-0) represented the ratio of gene expression level in 35S:HA-AtOFP8 transgenic plants to Col-0 in the data analysis. FC (Atofp $8-1$ vs Col-0) represented the gene in Atofp 8-1 mutant and Col-0 expression levels in the ratio. It was generally considered that there was no significant difference in gene expression in the range of $0.5-2.0$ for $\mathrm{FC}$ values, while genes with $\mathrm{FC}$ values greater than 2 or less than 0.5 were considered significant expression differences. There were 300 genes whose FC (35S:HA-AtOFP8 vs Col-0) value was more than 2 or less than 0.5, among which 192 genes were up-regulated, which were mainly related to photosynthesis, glucose metabolism, amino acid metabolism, fatty acid metabolism, alkanes and flavonoids biosynthesis (Fig. 5a). 108 genes were down-regulated, and these genes were associated with plant hormone signal transduction, tryptophan metabolism, nitrogen metabolism, endoplasmic reticulum protein processing and zeatin biosynthesis (Fig. 5b). There were 434 genes with FC (Atofp $8-1$ vs Col0 ) value more than 2 or less than 0.5, which 268 genes were up-regulated, mainly related to DNA replication, plant hormone signal transduction, glucose metabolism, photosynthesis, alkanes, pyrimidines and organisms alkaloid biosynthesis processes (Fig. 5c). 166 down-regulated genes were mainly associated with processes such as glyceride metabolism, endoplasmic reticulum protein processing, plant pathogen interactions, flavonoid biosynthesis, amino acid metabolism, and anthocyanin synthesis (Fig. 5d).

\section{The expression of genes related to epicuticular waxes varies greatly in 35S:HA-AtOFP8}

We chose some genes involved in epicuticular waxes synthesis and metabolism from gene chip results to test by qRT-PCR (Table 3). Results showed that the expression of KCS10 was up-regulated by more than tenfold in 35S:HA-AtOFP8 compared with Col-0. The expression of LTP4, FAR4 and GL2 also increased by more than 5 times in 35S:HA-AtOFP8 (Fig. 6a). In addition, the expression of CER4, ADH1 and At2g18370 was raised about twice in 35S:HA-AtOFP8, while significantly down-regulated in Atofp 8-1 compared with Col-0 (Fig. 6b). Contrary to these, the expression of CER2, At2g42990 and At1g06360 in 35S:HA-AtOFP8 was less than half of Col-0, while up-regulated in Atofp8-1 compared with Col-0 (Fig. 6c). These results show that AtOFP8 could change the expression of genes involving in epicuticular waxes accumulation, largely promoting the expression. 

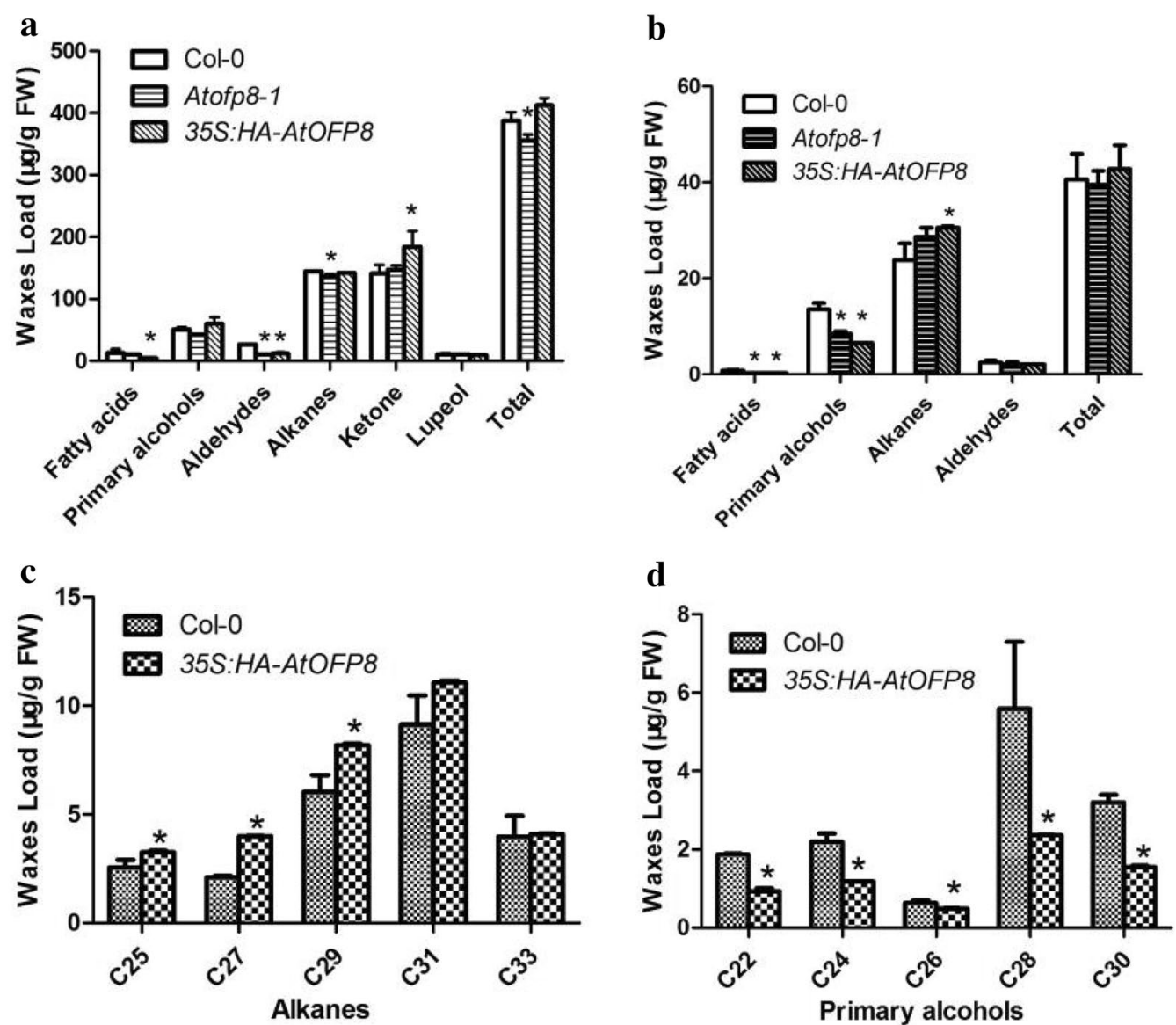

Fig. 4 Epicuticular waxes accumulation in the stems (a) and leaves (b-d). 5 weeks old stems and leaves were used. Biological triplicates, each with technical duplicates, were averaged. Statistical significance of the measurements was by comparing $\mathrm{Col}-0\left({ }^{*} \mathrm{P}<0.05\right)$. Bars indicate SE of the mean. FW fresh weight

\section{Cuticle transpiration slows in 35S:HA-AtOFP8}

After observing so many changes related to epicuticular waxes, we made water loss assays to test whether stratum corneum changed. Results showed that water loss in Col-0 was slower than that in Atofp 8-1, but faster than that in 35S:HA-AtOFP8 (Fig. 7a). Further chlorophyll leaching experiment showed that the percentage is the lowest in 35S:HA-AtOFP8 after $70 \mathrm{~min}$ (Fig. 7b). These results indirectly prove that there are more epicuticular waxes distributed on the leaves surface of 35S:HA-AtOFP8.

\section{Discussion}

To explore the function of AtOFP8, we designed this experiment using Atofp8-1, Col-0 and 35S:HA-AtOFP8. We analyzed microscopic distribution of epicuticular waxes, all kinds of component content, gene expression difference, which all results could demonstrate that AtOFP8 was able to regulate epicuticular waxes accumulation in some way. This laboratory finding provides a new direction for research AtOFP8 even AtOFPs.

Although the total amount of waxes in leaves was little difference, changes in specific components were obvious. Maybe AtOFP8 only changed the conversion of some certain components in leaves, so the total amount of waxes in leaves changed little. For example, in the 35S:HAAtOFP8 leaves, alkanes were significantly increased compared with the wild type plants, while primary alcohols were significantly reduced (Fig. 4c, d). Another reason was the presence of functional redundancy. Loss of AtOFP8 might induce expression of other genes that had the same function, so the content of epicuticular waxes in the Atofp 8-1 did not change significantly.

Due to diverse component of epicuticular waxes and its complex metabolic process, we do not determine what a role AtOFP8 plays in the metabolic process of waxes. In addition, functional redundancy exists in the transcription factors AtOFPs all the time, so it is difficult to find the its specific function invisibly. This is another 


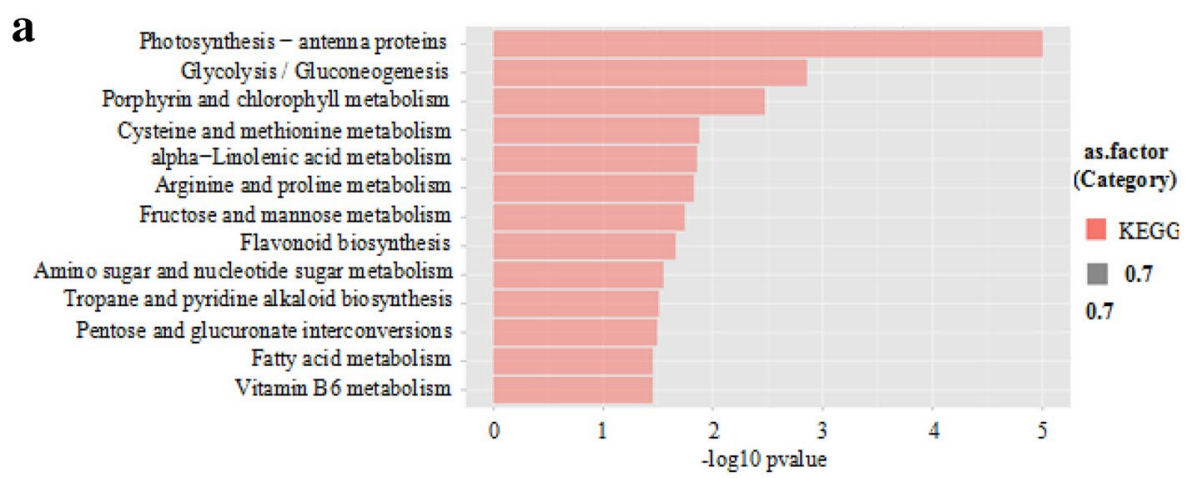

b

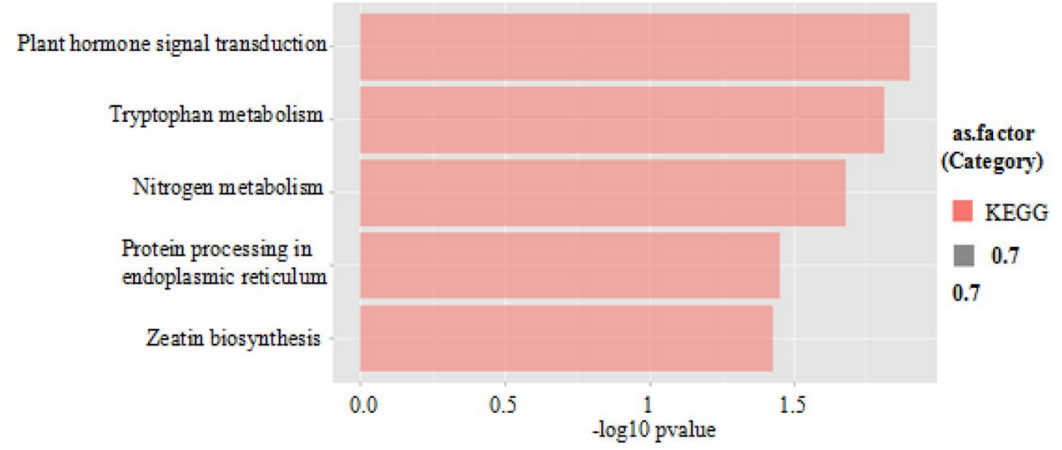

c DNA replication Plant hormone signal transduction Pentose and glucuronate interconversions Photosynthesis - antenna proteins

Tropane and pyridine alkaloid biosynthesis Nittogen metabolism Lysine biosynthesis Pyrimidine metabolism

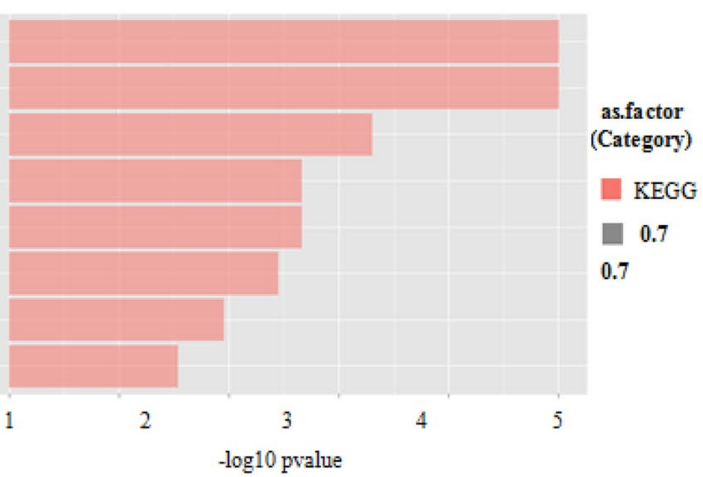

d

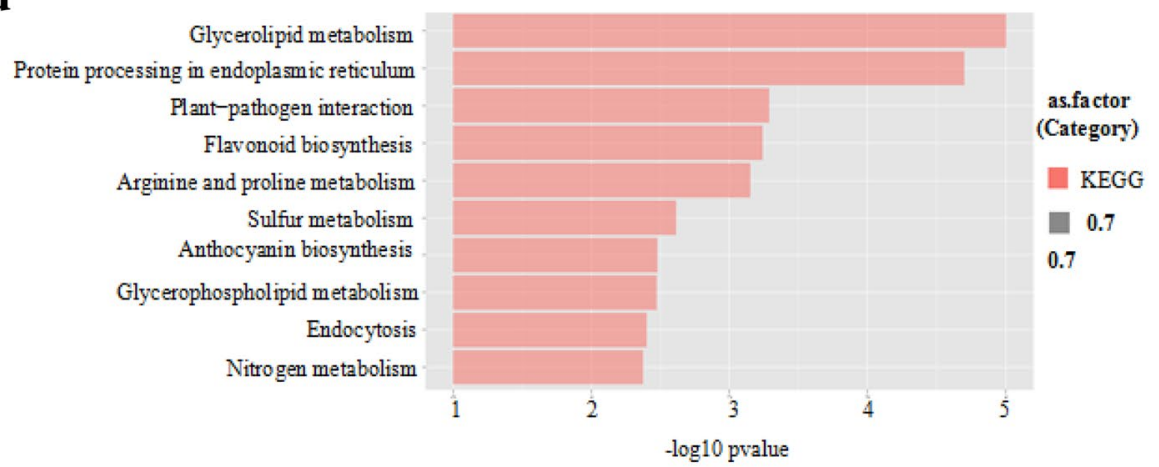

Fig. 5 Pathway enrichment affected by AtOFP8 gene. a Pathway enrichment of 35S:HA-AtOFP8 vs Col-0 up-regulated genes. b Pathway enrichment of 35S:HA-AtOFP8 vs Col-0 down-regulated genes. c Pathway enrichment of Atofp8-1 vs Col-0 up-regulated genes. d Pathway enrichment of Atofp81 vs Col-0 down-regulated genes 
Table 3 Genes about epicuticular waxes accumulation

\begin{tabular}{llcll}
\hline Gene & Symbol & FC in 35S:HA-AtOFP8 & FC in Atofp8-1 & Description \\
\hline At5g59310 & LTP4 & 4.6633 & 1.1343 & Lipid transfer protein 4 \\
At2g18370 & & 2.2816 & -1.7933 & Lipid transfer protein \\
At4g33790 & CER4 & 1.9469 & -1.2743 & Fatty acyl-CoA reductase \\
At1g04220 & KCS2 & 1.9174 & -1.0575 & 3-Ketoacyl-CoA synthase 2 \\
At1g77120 & ADH1 & 1.9057 & -2.2203 & Alcohol dehydrogenase 1 \\
At2g26250 & KCS10 & 1.8824 & -1.2770 & 3-Ketoacyl-CoA synthase 10 \\
At3g44540 & FAR4 & 1.8416 & -1.2542 & Fatty acyl-CoA reductase 4 \\
At1g79840 & GL2 & 1.6498 & -1.3211 & Lipid binding function \\
At5g42800 & DFR & 1.4015 & -2.5641 & Dihydroflavonol 4-reductase \\
At2g42990 & & -1.0988 & 2.9812 & Lipid catabolic process \\
At1g06360 & & -1.2762 & 1.7454 & Fatty acid desaturase family protein \\
At4g24510 & CER2 & -1.1792 & 1.4884 & Fatty acid elongation \\
\hline
\end{tabular}
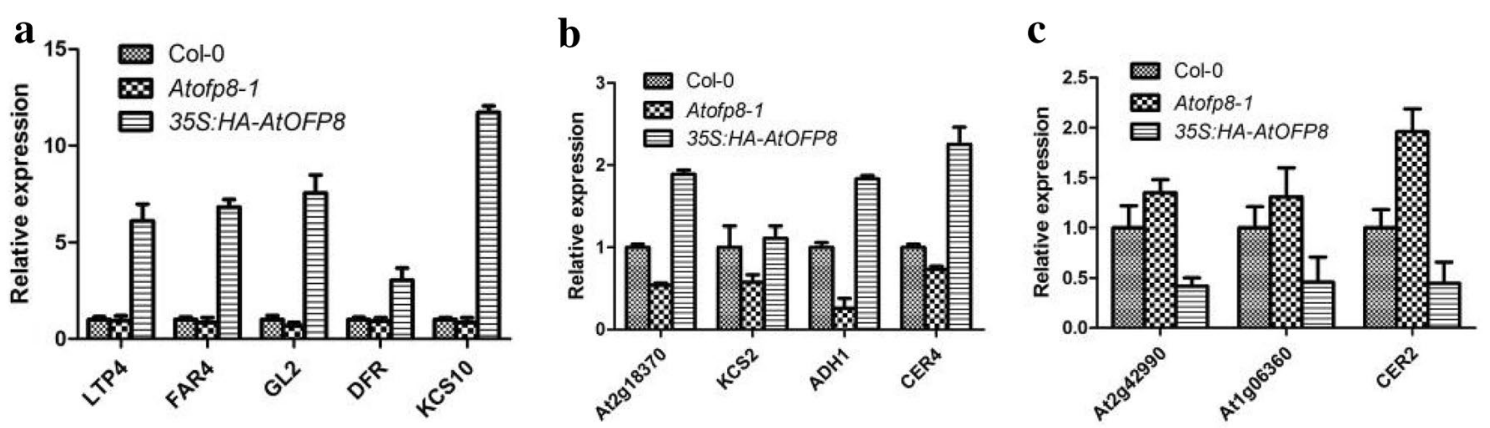

Fig. 6 Expression of genes that relate to waxes in Arabidopsis $(\mathbf{a}, \mathbf{b}, \mathbf{c})$. The leaves of 3 weeks old plants were used. Bars indicate SE of the mean
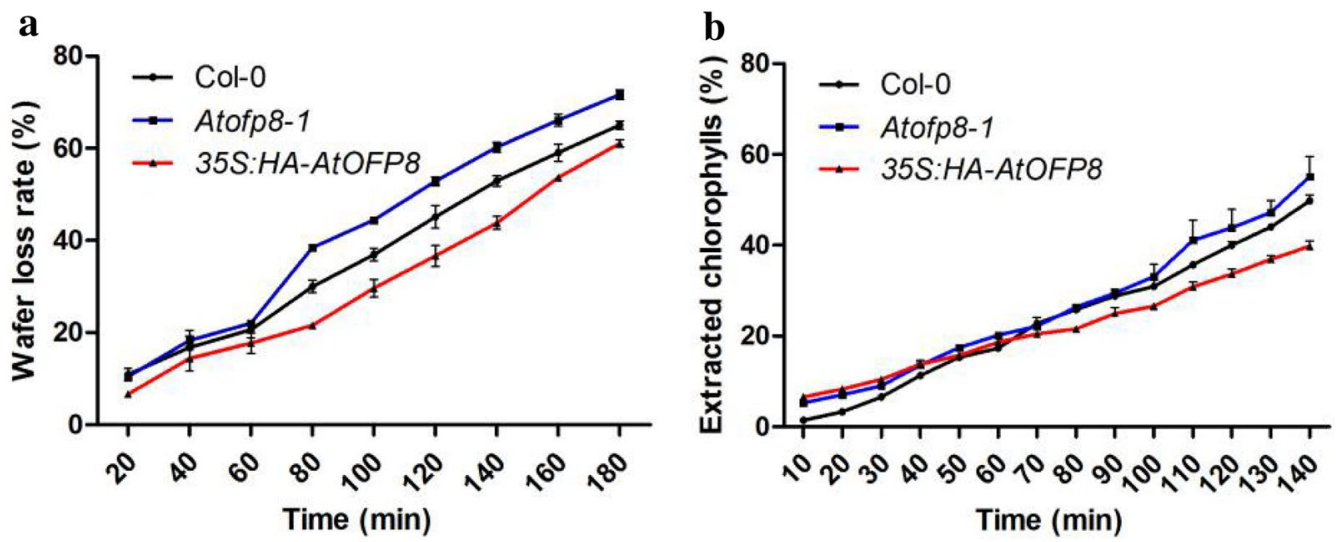

Fig. 7 Cuticular transpiration in Col-0, Atofp8-1 and 35S:HA-AtOFP8 leaves. 5 weeks old leaves were used. Bars indicate SE of the mean. a Water loss assays. b Chlorophyll leaching assays

reason that we cannot understand which part AtOFP8 affects epicuticular waxes accumulation. In spite of these, various aspects of changes of epicuticular waxes are obvious enough to prove that it plays an important role that AtOFP8 could change genes expression to affect epicuticular waxes accumulation. 


\section{Abbreviations}

AtOFP: Arabidopsis thaliana ovate family proteins; BSTFA: bis(trimethylsilyl) trifluoroacetamide; GC-MS: gas chromatography-mass spectrometer; MS: Murashige and Skoog; OFPs: ovate family proteins; SAS: SBC analysis system; VLCFAs: very long chain fatty acids.

\section{Authors' contributions}

YC designed the experiments. YT, WZ, YY, PF, HL and YC performed the experiments and analyzed the data. YT drafted the initial manuscript. All the authors agreed on the contents of the paper. All authors read and approved the final manuscript.

\section{Author details}

${ }^{1}$ Northeast Agricultural University, Harbin 150030, China. ${ }^{2}$ Keshan Branch of Heilongjiang Academy of Agricultural Sciences, Keshan 160000, Heilongjiang Province, China. ${ }^{3}$ College of Pharmacy, Qiqihar Medical University, Qiqihar 161006, China.

\section{Acknowledgements}

This work was supported by the National Natural Science Foundation of China (Project No. 30870144).

\section{Competing interests}

The authors declare that they have no competing interests.

\section{Availability of data and materials}

Not applicable.

\section{Consent for publication}

We agree to publish in this journal.

\section{Ethics approval and consent to participate}

This study does not violate ethical standards and all of us consent to participate.

\section{Funding}

This work was supported by the National Natural Science Foundation of China (Project No. 30870144)

\section{Publisher's Note}

Springer Nature remains neutral with regard to jurisdictional claims in published maps and institutional affiliations.

Received: 8 December 2017 Accepted: 16 April 2018

Published online: 24 April 2018

\section{References}

Arondel V, Vergnolle C, Cantrel C, Kader JC (2000) Lipid transfer proteins are encoded by a small multigene family in Arabidopsis thaliana. Plant Sci 157:1-12
Domergue F, Vishwanath SJ, Joubes J, Ono J, Lee JA, Bourdon M, Alhattab R, Lowe C, Pascal S, Lessire R, Rowland O (2010) Three Arabidopsis fatty acylcoenzyme A reductases, FAR1, FAR4, and FAR5, generate primary fatty alcohols associated with suberin deposition. Plant Physiol 153:1539-1554

Go YS, Kim H, Kim HJ, Suh MC (2014) Arabidopsis cuticular wax biosynthesis is negatively regulated by the DEWAX gene encoding an AP2/ERF-type transcription factor. Plant Cell 26:1666-1680

Hackbusch J, Richter K, Muller J, Salamini F, Uhrig JF (2005) A central role of Arabidopsis thaliana ovate family proteins in networking and subcellular localization of 3-aa loop extension homeodomain proteins. Proc Natl Acad Sci USA 102:4908-4912

Holmes MG, Keiller DR (2002) Effects of pubescence and waxes on the reflectance of leaves in the ultraviolet and photosynthetic wavebands: a comparison of a range of species. Plant Cell Environ 25:85-93

Koch K, Ensikat HJ (2008) The hydrophobic coatings of plant surfaces: epicuticular wax crystals and their morphologies, crystallinity and molecular self-assembly. Micron 39:759-772

Li EY, Wang SC, Liu YY, Chen JG, Douglas CJ (2011) Ovate family protein 4 (OFP4) interaction with KNAT7 regulates secondary cell wall formation in Arabidopsis thaliana. Plant J 67:328-341

Liu JP, Eck VJ, Cong B, Tanksley SD (2002) A new class of regulatory genes underlying the cause of pear-shaped tomato fruit. Proc Natl Acad Sci USA 99:13302-13306

Liu D, Sun W, Yuan YW, Zhang N, Hayward A, Liu YL, Wang Y (2014) Phylogenetic analyses provide the first insights into the evolution of OVATE family proteins in land plants. Ann Bot 113:1219-1233

Lolle SJ, Hsu W, Pruitt RE (1998) Genetic analysis of organ fusion in Arabidopsis thaliana. Genetics 149:607-619

Pagnussat GC, Yu HJ, Sundaresan V (2007) Cell-fate switch of synergid to egg cell in Arabidopsis eostre mutant embryo sacs arises from misexpression of the BEL1-like homeodomain gene BLH1. Plant Cell 19:3578-3592

Rowland O, Zheng HQ, Hepworth SR, Lam P, Jetter R, Kunst L (2006) CER4 encodes an alcohol-forming fatty acylcoenzyme A reductase involved in cuticular wax production in Arabidopsis. Plant Physiol 142:866-877

Shepherd T, Griffiths DW (2006) The effects of stress on plant cuticular waxes. New Phytol 171:469-499

Sieber P, Schorderer M, Ryser U, Buchala A, Kolattukudy P, Metraux JP, Nawrath C (2000) Transgenic Arabidopsis plants expressing a fungal cutinase show alterations in the structure and properties of the cuticle and postgenital organ fusions. Plant Cell 12:721-738

Wang SC, Chang Y, Guo JJ, Chen JG (2007) Arabidopsis ovate family protein 1 is a transcriptional repressor that suppresses cell elongation. Plant J $50: 858-872$

Wang SC, Chang Y, Guo JJ, Zeng QN, Ellis BE, Chen JG (2011) Arabidopsis Ovate Family Proteins, a novel transcriptional repressor family, control multiple aspects of plant growth and development. PLOS ONE 6:e23896

Xia Y, Nikolau BJ, Schnable PS (1996) Cloning and characterization of CER2, an Arabidopsis gene that affects cuticular wax accumulation. Plant Cell 8:1291-1304

\section{Submit your manuscript to a SpringerOpen ${ }^{\circ}$ journal and benefit from:}

- Convenient online submission

- Rigorous peer review

- Open access: articles freely available online

- High visibility within the field

- Retaining the copyright to your article

Submit your next manuscript at $>$ springeropen.com 